Mai 1992

\title{
EXISTENCE OF EQUILIBRIA \\ IN THE OVERLAPPING GENERATIONS MODEL \\ THE NONTRANSITIVE CASE
}

\author{
Imed CHERIF* \\ Messaoud DEGHDAK" * \\ Monique FLORENZANO"**
}

$N^{\circ} 9205$

* CEPREMAP, CERMSEM (University of Paris I)

** University of Constantine

*** CNRS, CEPREMAP, CERMSEM (University of Paris I).

CEPREMAP : 142 rue du Chevaleret, 75013 PARIS, France. 


\section{EXISTENCE OF EQUILIBRIA IN THE OVERLAPPING GENERATIONS MODEL THE NONTRANSITIVE CASE}

\section{Résumé}

Ce papier traite de l'existence de l'équilibre général dans un modèle dynamique d'échange avec une double infinité dénombrable de périodes et d'agents. L'espace des biens considéré à chaque période est éventuellement de dimension infinie. Les préférences des agents ne sont supposées ni transitives ni totales.

Pour le modèle général où certains consommateurs peuvent avoir, comme chez Wilson, une durée de vie infinie, un premier théorème d'existence est démontré sous $I$ 'hypothèse classique qu'il existe un ensemble fini d'agents non négligeables.

Dans le cas particulier du modèle à générations, un deuxième théorème montre l'existence d'un équilibre Walrasien dans le cas standard où les agents $n$ 'ont de ressources que pendant leur durée de vie (finie) et l'existence d'un équilibre avec transferts positifs ou nuls si les agents détiennent des actifs réels sur une infinité de périodes. Dans ce dernier cas, l'existence $d$ 'un équilibre Walrasien est toutefois démontrée sous 1 'hypothèse qu'un ensemble fini d'agents détient une fraction positive du total des actifs réels à durée de vie infinie.

NOMENCLATURE JEL : 021, 111.

\section{Abstract}

This paper investigates the existence of competitive equilibria in dynamic exchange models with countably many periods and countably many agents. At each period the commodity space can be finite or infinite dimensional. The preference of agents are not assumed to be transitive or complete.

A first equilibrium existence theorem is established under the classical assumption that there exists a finite set of non-negligible agents.

In the particular case of an overlapping generations model, a second existence theorem allows simultaneously for finite-lived assets and infinite-lived assets and limits the previous assumption to infinite-lived assets. This theorem covers obviously the standard case of an overlapping generations model where the agents have no endowment outside their lifetime.

KEY-WORDS : Locally convex-solid topological vector lattices - Symmetric Riesz dual systems - Overlapping generations exchange model - Equilibrium Uniformly proper preferences. 
EXISTENCE OF EQUILIBRIA IN THE OVERLAPPING GENERATIONS MODEL THE NONTRANSITIVE CASE

Imed CHERIF - CEPREMAP and CERMSEM (University of Paris I)

Messaoud DEGHDAK - University of Constantine

Monique FLORENZANO - CNRS, CEPREMAP and CERMSEM (University of Paris I)

Summary. This paper investigates the existence of competitive equilibria in dynamic exchange models with countably many periods and countably many agents. At each period the commodity space can be finite or infinite dimensional. The preferences of agents are not assumed to be transitive or complete. A first equilibrium existence theorem is established under the classical assumption that there exists a finite set of non-negligible agents. In the particular case of an overlapping generations model, a second existence theorem allows simultaneously for finite-lived assets and infinite-lived assets and limits the previous assumption to infinite-lived assets. This theorem covers obviously the standard case of an overlapping generations model where the agents have no endowment outside their lifetime.

\section{Introduction}

This paper deals with the existence of equilibria in dynamic exchange models with a countable infinity of discrete time periods and agents. Overlapping generations models are a special case of such models. They are defined here by the double condition that each agent can consume only during a finite set of periods, identified with its lifetime, and that at each period only a finite set of agents are alive.

As in Aliprantis et al. [1,2], the commodity space at each period may be infinite dimensional. This possibility allows for a consideration of stochastic dynamic models with an infinite dimensional state space at each period and an overlapping generations analysis of financial markets. The other assumptions on the economy are comparable with or weaker than those used in Wilson [21] and Burke [11] in a finite dimensional framework. In particular, unlike Balasko and Shell [7], Balasko et al. [6], Aliprantis et al. $[1,2]$, Geanakoplos and Polemarchakis [16], the preferences of agents 
are not assumed to be transitive or complete on their consumption set and we explicitely include both overlapping generations of finite-lived agents and infinite-lived agents.

The existence of equilibria is first established under the classical assumption that there exists a finite set of non-negligible agents, in the sense that a finite set of agents owns a positive fraction of the total (social) endowment. As it is well known, the value of the social endowment at the equilibrium prices is finite and such equilibria are Pareto-optimal.

Without this assumption, by perturbing the initial individual endowments so as to satisfy the previous assumption and by passing to limit on the equilibria of a suitable sequence of perturbed economies, we prove for overlapping generations models an existence result comparable with those of Wilson or Burke : there exists an attainable allocation and a price such that each agent endowed only with finite-lived assets optimizes its preferences under its budget constraint, while the other agents perform the same optimization under a revenue constraint which may exceed the value of their initial endowment. Since Samuelson [20], the non-negative monetary transfers needed at equilibrium by agents endowed with infinite-lived assets are interpreted as fiat money. The existence of Walrasian equilibria in a standard overlapping generations model where the agents have no endowment outside their finite lifetime is obviously a particular case of this general result. The equilibrium price does not necessarily give a finite value to the total endowment and equilibria as well as Samuelson-type transfer equilibria may fail to be Pareto-optimal.

Dynamic models with a finite set of non-negligible agents and overlapping generations models with only finite-lived assets are two extreme case where equilibrium is generally claimed to exist. One can think of natural intermediate cases for which the two assumption are both inappropriate. In order to get a wide variety of economic interpretations, we assume in this paper the existence of two kinds of assets, limit to the first kind the assumption of a finite set of non-negligible agents, assume that the assets of the second kind are always finite-lived and prove under a (strong) additional assumption the existence of a Walrasian equilibrium not necessarily Pareto-optimal.

To sum up, this paper proves in the framework of Aliprantis et al, existence results comparable with those got by Wilson and Burke in the finite dimensional framework. Beyond this double extension, this paper 
gives a first analysis of the equilibrium existence problem for overlapping generations model with durable and non-durable "goods".

Our method of proof derives from the arguments of Bewley [10], Richard and Srivastava [19], Aliprantis et al.[1, 2], Burke [11]. Our main innovation is the construction of a commodity space for the whole economy which allows to treat of as well the first case of a dynamic economy with a finite set of non-negligible agents as the case of an overlapping generations model without this assumption. This construction differs from that of Aliprantis et al. who construct a specific commodity space for overlapping generations models. It uses Köthe-type spaces, first introduced by Besada et al. [8] for modelling intertemporal equilibrium.

The paper is organized as follows. In Section 2, we precise the model and its assumptions. In Section 3, we give the definition and the properties of the commodity space. In Sections 4,5 and 6 , we prove the existence of equilibria under different sets of assumptions on the preferences and endowments of agents.

\section{The economic model}

We consider in this paper a dynamic model of pure exchange with countably many discrete time periods and countably many agents; overlapping generations models are particular instances of this general model. Let $N$ be the set of consumers and, for each consumer $i$, let $X^{i}$ be his consumption set, $P^{i}$ his preference correspondence over $X^{i}, \omega^{i}$ his endowment and $T^{i}$ the collection of his lifetime periods. We define an economy as a list $\left(X^{i}, P^{i}, \omega^{i}, T^{i}\right)_{i \in N}$ for which we set the following assumptions which generalize, in an infinite dimensional setting, some standard assumptions used in the finite dimensional case for dynamic models of exchange without ordered preferences :

1. For each period $t$, the commodity-price duality is represented by a symmetric Riesz dual system $\left\langle E_{t}, E_{t}\right\rangle . E_{t}$ and $E_{t}$ are respectively the commodity space and the price space at period $t$. The value of $x_{t} \in E_{t}$ at prices $p_{t} \in E_{t}^{\prime}$ is denoted by $p_{t} \cdot x_{t}=\left\langle x_{t}, p_{t}\right\rangle$.

2 . $N$, the set of consumers, is countably infintte (for simplicity, $N$ is assumed to be the set of natural integers).

For overlapping generations models, Assumption 2 is replaced by: $2^{\prime}$. For each $i, T^{i}$ is finite and for each $t$, the set $\left\{t \in N \mid t \in T^{i}\right\}$ ts 
finite.

In both cases, it is implicitely assumed that at least two consumers are alive at each period.

3. For each consumer $i$, there exists $a^{i} \in \prod_{t \in T^{i}} E_{t}^{+}$such that $x^{i}=\left\{a^{i}\right\}+\prod E_{t}^{+}$. Every agent consumes only during his lifetime and, at $t \in T^{i}$

each lifetime period, may consume any bundle besides some survival consumption.

We will indicate later how to weaken this assumption on the stucture of the individual consumption sets.

4. For each consumer $i, \omega^{i}>0$ in $\prod_{t=1}^{\infty} E_{t}^{+}$with $a^{i} \leq\left(1-\lambda^{i}\right) \omega^{i}$ for some $\lambda^{i}$ : $0<\lambda^{i}<1$. Note that, with this assumption, the agents may have endowments outside their lifetime. For economic interpretation, an initial endowment must be thought of as a real asset, owned by the consumer at his birthdate, the value of which depends on prices.

We also assume that the total endowment is well-defined, i.e. that $\omega=$ $\sup \left\{\sum_{i=1}^{n} \omega^{i} ; n=1,2, \ldots\right\}$ exists in $\prod_{t=1}^{\infty} E_{t}$. We say that an allocation $x \in \prod_{i \in N} x^{i}$ is attainable if $\sup \left\{\sum_{i=1}^{n} x^{i} ; n=1,2, \ldots\right\}=\omega$. This is also noted by $\sum_{i=1}^{n} x^{i} \uparrow \omega . \hat{\mathrm{x}}$ will denote the set of the attainable allocations for the economy $\mathscr{E}$.

5. For each $i, P^{i}: X^{i} \mapsto X^{i}$ has open lower sections if $X^{i}$ is endowed with the suitable product of the $\sigma\left(E_{t}, E_{t}\right)$-topologies, open values if $x^{i}$ is endowed with the suitable product of the absolute weak topologies $|\sigma|\left(E_{t}, E_{t}^{\prime}\right)$.

For every $x^{i} \in X^{i}, x^{i} \notin$ co $P^{i}\left(x^{i}\right)$. Furthermore, for all $x^{i}, y^{i} \in X^{i}$, $x^{i} \geq y^{i}$ implies $P^{i}\left(x^{i}\right) \subset P^{i}\left(y^{i}\right)$ and $\left(P^{i}\right)^{-1}\left(y^{i}\right) \subset\left(P^{i}\right)^{-1}\left(x^{i}\right)$.

The first part of Assumption 5 is a standard continuity assumption on preferences which has interpretations in terms of impatience relatively to future events and of indifference, at each period, relatively to events of low probability. Besides a standard convexity requirement, the last part corresponds to monotonicity when each $P^{i}$ is the asymmetric part of a total 
preorder on $X^{i}$.

6. The total endowment $\omega$ of the economy is desirable for every consumer in every component of an attainable allocation: if $x \in \hat{x}$, then for each $i$ and for every $\alpha>0$ there exists $z^{i} \in \prod_{t \in T^{i}} E_{t}^{+}, z^{i} \leq \alpha \omega$ such that $x^{i}+z^{i} \in P^{i}\left(x^{i}\right)$.

This assumption implies in particular local non-satiation of preferences in every component of an attainable allocation, whatever be the locallysolid topology considered on $\prod_{t=1}^{\infty} E_{t}$.

7. If $x$ is an attainable allocation and $I$ any proper and nonempty subset of $N$. there exist $i \in I$ and $z^{i} \in \prod_{t \in T^{i}} E_{t}^{+}$such that $z^{i} \leq \sum_{j \notin I} \omega^{j}$ and $x^{i}+z^{i} \in P^{i}\left(x^{i}\right)$.

Assumption 7 is known as an irreducibility condition. It requires that the preferences and endowments of agents be sufficiently interconnected. It should be remarked that, in view of Assumptions 1 and 5, there is no loss of generality to assume in 7 that if $x$ is an attainable allocation and $I$ any proper and non-empty subset of $N$, there exist $i \in I, z^{i} \in \prod_{t \in T^{i}} E_{t}^{+}$and a finite subset $F$ of $N \backslash I$ such that $z^{i} \leq \sum_{j \in F} \omega^{j}$ and $x^{i}+z^{i} \in P^{i}\left(x^{i}\right)$.

The next assumption is a strong version of the strict positivity at each period of the aggregate endowment :

8. For every period $t, \omega_{t}$, the total endowment at the period $t$, is an order-unit of $E_{t}$ and $E_{t}=\left(E_{t}\right)_{n}^{\sim}$ (the order-contimuous dual of $E_{t}$ ).

$\left\langle\mathbb{R}^{l} t, \mathbb{R}^{2}\right\rangle$ with $\omega_{t}>0,\left\langle L_{\infty}(\mu), L_{1}(\mu)\right\rangle, \mu$-finite, with $\omega_{t}$ equal to a constant mapping, are typical examples of symmetric Riesz dual systems satisfying this assumption. Assumption 8 will be weakened in the last section.

As we will see later, the above assumptions (or a slightly strengthened version of some of them) allow to prove the existence of a very weak equilibrium notion, called as in Aliprantis et al. [1,2] weak quasiequilibrium. To get an equilibrium existence result, we need some assumption beyond 1-8. We give there two versions of such an additional assumption: 
9. A finite subset of agents owns a positive fraction of the total endowment i.e. there exist $I_{0} \subset N, I_{0}$ finite and $\theta>0$ such that $\sum_{i \in I_{0}} \omega^{i} \geq \theta \omega$

Given 8 , the assumption 9 clearly rules out the standard overlapping generations model where each consumer has no endowment outside his finite lifetime. According to a suggestion from T.C. Bergstrom [9], in Assumption 10, we allow simultaneously for finite-lived and infinite-lived assets and basically limit Assumption 9 to infinite-lived assets.

10. For each period $t, E_{t}=F_{t} \oplus G_{t}$. If $\omega^{i}=\omega^{\prime i}+\omega^{\prime \prime}$ with $\omega_{t}^{i} \in F_{t}^{+}$and $\omega_{t}^{\prime \prime} \in G_{t}^{+}$, then

- for each $i, \omega^{n i} \in \prod_{t \in T^{i}} G_{t}^{+}$and

- there exist $I_{0} \subset N, I_{0}$ finite and $\theta>0$ such that $\sum_{i \in I_{0}} \omega^{\prime i} \geq \theta \omega^{\prime}$.

Assumption 9 is obviously a particular case of Assumption 10. Assumption 10 is also satisfied in a standard overlapping generations model with only finite-lived assets. Besides these two extreme cases, the main interest of Assumption 10 is to allow for economies in which there are simultaneously infinitely durable and non-durable "goods". The consumers are assumed to have no endowment in non-durable goods outside their lifetime, while a finite set of individuals own a non-negligible fraction of the aggregate endowment of infinitely durable goods.

\section{Construction of the commodity-price duality}

In this section, we construct the Riesz dual system that defines the commodity-price duality for our dynamic model. This construction differs from that of Aliprantis et al. [1, 2] in its motivation and its mathematical background. It uses Köthe's perfect spaces (see [17] $\$ 30$ ), first introduced by Besada et al. [8] as commodity and price spaces which arise in a natural way in connection with the initial endowments of the agents, for a model with countably many time periods.

Let us restrict the price space of the entire economy to

$$
P=\left\{p \in \prod_{t=1}^{\infty} E_{t}\left|\sum_{t=1}^{\infty}\right| p_{t} \mid \cdot \omega_{t}^{i}<+\infty, \forall i \in N\right\} .
$$


For symmetry, let us define

$$
\Lambda(P)=\left\{x \in \prod_{t=1}^{\infty} E_{t}\left|\sum_{t=1}^{\infty}\right| p_{t}|.| x_{t} \mid<+\infty, \forall p \in P\right\}
$$

which may be thought to be the commodity space of the economy.

The commodity-price duality of the model is given by the bilinear form : $\langle x, p\rangle=p \cdot x=\sum_{t=1}^{\infty} p_{t} \cdot x_{t}$. If $x \in \Lambda(P)$ and $p \in P, p . x$ is the value of $x$ reckoned at the prices $p=\left(p_{t}\right)$.

Let $\tau$ denote on $\Lambda(P)$ the topology generated by the collection $\left\{\rho_{p} ; p \in P\right\}$ of seminorms defined on $\wedge(P)$ by $: \rho_{p}(x)=\sum_{t=1}^{\infty}\left|p_{t}\right| \cdot\left|x_{t}\right|$. Symmetrically, let $\tau^{\prime}$ denote on $P$ the topology generated by the collection $\left\{\rho_{x} ; x \in \Lambda(P)\right\}$ of seminorms defined on $P$ by $: \rho_{x}(p)=\sum_{t=1}^{\infty}\left|p_{t}\right| \cdot\left|x_{t}\right|$.

Proposition 3.1. $\langle\Lambda(P), P\rangle$ is a dual pair. $T$ (resp. $T^{\prime}$ ) is a Hausdorff locally convex-solid topology consistent with this duality.

Proof. Let $\Phi$ (resp. $\Phi^{\prime}$ ) denote the set of all elements of $\prod_{t=1}^{\infty} E_{t}$ (resp. $\prod_{t=1}^{\infty} E_{t}^{\prime}$ ) with only finitely many non-zero coordinates. Obviously $\Lambda(P)$ and $P$ are Riesz spaces, more precisely ideals of $\prod_{t=1}^{\infty} E_{t}$ and $\prod_{t=1}^{\infty} E_{t}$ containing $\Phi$ and $\Phi^{\prime}$. The separations properties of the bilinear form $\langle x, p\rangle=p \cdot x=\sum_{t=1}^{\infty} p_{t} \cdot x_{t}$ follow from the last remark. Each seminorm $\rho_{p}(x)$ (resp. $\left.\rho_{x}(p)\right)=$ $\sum_{t=1}^{\infty}\left|p_{t}\right| .\left|x_{t}\right|$ is obviously monotone, i.e. a Riesz seminorm ||$x|\leq| y \mid \Rightarrow$ $\rho_{p}(x) \leq \rho_{p}(y) \quad$ (resp. $|p| \leq|q| \Rightarrow \rho_{x}(p) \leq \rho_{x}(q)$ ); thus $\tau$ (resp. $\tau^{\prime}$ ) is a locally convex-solid topology on $\Lambda(P)$ (resp. P).

It should be clear that $P \subset(\Lambda(P), \tau)^{\prime}$, the topological dual of $\Lambda(P)$. To prove the converse inclusion, let us remark that on each factor $E_{t}, \tau$ coincides with the absolute weak topology $|\sigma|\left(E_{t}, E_{t}^{\prime}\right)$ generated by the seminorms : $x_{t} \rightarrow\left|p_{t}\right| \cdot\left|x_{t}\right|$. Hence, if $f \in(\Lambda(P), \tau)^{\prime}$, for each there exists $p_{t} \in E_{t}^{\prime}$ such that $f\left(0, \ldots, 0, x_{t}, 0, \ldots\right)=p_{t} \cdot x_{t}$. Since for all $x$ in $\Lambda(P)$, $\left(x_{1}, x_{2}, \ldots, x_{t}, 0, \ldots\right) \tau$-converges to $x, f(x)=\sum_{t=1}^{\infty} p_{t}, x_{t}$. It remains to prove that $p=\left(p_{t}\right) \in P .|f|\left(0, \ldots, 0, x_{t}, 0, \ldots\right)=\left|p_{t}\right|, x_{t}$, so that for every $t$, 
$|f|\left(\omega^{i}\right)=\sum_{t=1}^{\infty}\left|p_{t}\right| \cdot \omega_{t}^{i}<+\infty$. From a similar proof, it follows that $\Lambda(P)=$ $\left(P, T^{\prime}\right)^{\prime}$. Finally, if $\sigma(\Lambda(P), P)$ and $\sigma(P, \Lambda(p))$ are the weak topologies associated with the duality $\langle\Lambda(P), P\rangle$, remark that $\sigma(\Lambda(P), P) \subset \tau$ and $\sigma(P, \Lambda(P)) \subset \tau^{\prime}$, which proves that $\tau$ and $\tau^{\prime}$ are Hausdorff.

口

Proposition 3.2. Each order-interval of $\wedge(P)$ (resp. $P$ ) is $\sigma(\Lambda(P), P)$ (resp. $\sigma(P, \wedge(P))$-compact.

Proof. If $u>0$ in $\Lambda(P)$, let $[-u,+u]$ be an order-interval of $\Lambda(P)$. For any $\varepsilon>0, \rho_{u}(p)<\varepsilon$ and $x \in[-u,+u]$ imply

$$
\text { । } \sum_{t=1}^{\infty} p_{t} \cdot x_{t}\left|\leq \sum_{t=1}^{\infty}\right| p_{t} \cdot x_{t}\left|\leq \sum_{t=1}^{\infty}\right| p_{t}|\cdot| x_{t} \mid \leq \rho_{u}(p)<\varepsilon .
$$

This shows the $\tau^{\prime}$-equicontinuity of $[-u,+u]$. The $\sigma(\wedge(P), P)$-relative compactness of $[-u,+u]$ follows from the Alaoglu-Bourbaki theorem. Recall now that each $\left\langle E_{t}, E_{t}^{\prime}\right\rangle$ is a symmetric Riesz dual system. Each $\left[-u_{t},+u_{t}\right]$ is $\sigma\left(E_{t}, E_{t}^{\prime}\right)$-compact. It follows easily that $[-u,+u]$ is $\sigma(\wedge(P), P)$-closed.

The $\sigma(P, \wedge(p))$-compactness of order-intervals of $P$ is proved in a similar way.

\section{口}

Applying Theorem 22.1 of Aliprantis and Burkinshaw [3], we see that $\wedge(P)$ (resp. $P$ ) is Dedekind-complete and $\tau$ (resp. $\tau^{\prime}$ ) is a Lebesgue topology. In other words, $\langle\Lambda(P), P\rangle$ is a symmetric Riesz dual system. If $(\Lambda(P))_{n}$ (resp. $P_{n}^{\sim}$ ) denotes the collection of all order-continuous, order-bounded linear forms on $\Lambda(P)$ (resp. P), $P \subset(\Lambda(P))_{n}^{\sim}$ (resp. $\left.\wedge(P) \subset P_{n}^{\sim}\right)$.

Under Assumption 10,

$$
P=\left\{p \in \prod_{t=1}^{\infty} E_{t}\left|\sum_{t=1}^{\infty}\right| p_{t} \mid \cdot \omega_{t}^{i}<+\infty\right\}
$$

and $\omega^{\prime} \in \Lambda(P)$. In the overlapping generations model with only finite-lived assets, $\omega^{\prime}=0$,

$P=\prod_{t=1}^{\infty} E_{t}$ and $\wedge(P)=\Phi=\left\{x \in \prod_{t=1}^{\infty} E_{t} \mid \exists k \geq 1, x_{t}=0 \forall t \geq k\right\}$.

If the economy satisfies Assumption 9, then

$$
P=\left\{p \in E_{t}^{\prime} \quad\left|\prod_{t=1}^{\infty}\right| p_{t} \mid \cdot \omega_{t}<+\infty\right\}
$$

and $\omega \in \Lambda(P)$. This allows to define the principal ideal $A_{\omega}$ in $\wedge(P)$ generated by $\omega, A_{\omega}=\{x \in \Lambda(P) \quad|\exists \lambda>0| x \mid, \leq \lambda \omega\}$. Endowed with the 
Riesz norm : $\|x\|_{\omega}=\inf \{\lambda>0|| x \mid \leq \lambda \omega\}, A_{\omega}$ is a Banach lattice. Furthermore, $A_{\omega}$ is an AM-space with unit $\omega$. The norm dual of $\left(A_{\omega},\|.\|_{\omega}\right)$ will be denoted by $A_{\omega} ;$ it coincides with the order dual $A_{\omega}^{\sim}$ of $A_{\omega}$. Remark that, from the inequality $|x| \leq\|x\|_{\omega} \omega \forall x \in A_{\omega},\left\{x \in A_{\omega} \mid\|x\|_{\omega} \leq 1\right\}=$ $[-\omega,+\omega]$ and so $\omega$ belongs to the $\|.\|_{\omega}$ - interior of $A_{\omega}^{+}$. It is easily seen that $\left\langle A_{\omega}, A_{\dot{\omega}}\right\rangle$ is a Riesz dual system but not a symmetric one.

The next proposition presents useful properties of this system.

Proposition 3.3. Under the assumptions 1 and 8 , if $\omega \in \wedge(P), A_{\omega}$ is order dense in $\Lambda(P)$ and

$$
\left(A_{\omega}\right)_{n}^{\sim} \subset P \subset(\Lambda(P)) \tilde{n}_{n} \subset\left(A_{\omega}\right) \tilde{n}
$$

Proof. From the first part of the assumption 8, it follows that each factor $E_{t}$ can be considered as a Riesz subspace of $A_{\omega}$. For all $x$ in $\wedge(P)$. $\left(x_{1}, x_{2}, \ldots, x_{t}, 0, \ldots\right) \uparrow x$. This proves the first part of the statement.

To prove the first inclusion, let $f$ be any element of $\left(A_{\omega}\right)_{n}$. From the second part of the assumption 8 , one deduces that there exists $p \in\left(E_{t}\right)_{n}^{\sim}$ $=E_{t}^{\prime}$ such that $f(x)=\sum_{t=1}^{\infty} p_{t} \cdot x_{t}$ and that $p=\left(p_{t}\right) \in \mathrm{P}$.

The second inclusion was proved above; the third one is obvious.

Throughout this paper, we will use inclusion relations between the different topologies defined on $\Lambda(P)$. The topology $r$ defined on $\Lambda(P)$ is obviously finer than the weak topology $\sigma(\Lambda(P), P)$ which is also finer than the product of the weak topologies $\sigma\left(E_{t}, E_{t}^{\prime}\right)$. Furthermore, the product of the weak topologies $\sigma\left(E_{t}, E_{t}\right)$ and $\sigma(\Lambda(P), P)$ coincide on $X^{i}$ (considered as a subset of $\Lambda(P))$, if $T^{i}$ is finite, and on every order-interval of $\Lambda(P)$. In the same way, the topology $\tau$ is finer than the product of the absolute weak topologies $|\sigma|\left(E_{t}, E_{t}\right)$ on $\Lambda(P)$ and coincides with it on $X^{i}$, if $T^{i}$ is finite, and on every order-interval of $\Lambda(P)$.

If $\omega \in \Lambda(P)$, in view of Proposition 3.3, $\sigma(\Lambda(P), P)$ is coarser on $A_{\omega}$ than $\sigma\left(A_{\omega}, A_{\omega}^{\prime}\right)$ which is also coarser than the $\|.\|_{\omega^{-}}$topology of $A_{\omega}$.

The equilibrium concepts are defined in relation with the commodity-price duality considered for the model.

An equilibrium (resp. quastequilibrium) with respect to the symetric Riesz dual system $\langle\Lambda(P), P\rangle$ consists of a price $\overline{\mathrm{p}} \in P, \overline{\mathrm{p}} \neq 0$ and an 
attainable allocation $\bar{x} \in \prod_{i \in N}\left(X^{i} \cap \wedge(P)\right)$ such that $\bar{p} \cdot \bar{x}^{i} \leq \bar{p} \cdot \omega^{i}$ holds for every $t \in N$ and that $x^{i} \in X^{i} \cap \wedge(P)$ and $x^{i} \in P^{i}\left(\bar{x}^{i}\right)$ imply $\overline{\mathrm{p}} \cdot x^{i}>\bar{p} \cdot \omega^{i}$ (resp. $\overline{\mathrm{p}} \cdot x^{i} \geq \bar{p} \cdot \omega^{i}$ ).

A weak quasiequilibrium with respect to the symmetric Riesz dual system $\langle\Lambda(P), P\rangle$ consists of a price $\bar{p} \in P, \bar{p} \neq 0$ and an attainable allocation $\bar{x} \in \prod_{i \in N}\left(X^{i} \cap \wedge(P)\right) \quad$ such that $x^{i} \in X^{i} \cap \wedge(P)$ and $x^{i} \in p^{i}\left(\bar{x}^{i}\right)$ imply $\overline{\mathrm{p}} \cdot x^{i} \geq \bar{p} \cdot \omega^{i}$.

If $\omega \in \Lambda(P)$, the same equilibrium concepts with respect to the Riesz dual system $\left\langle A_{\omega}, A_{\omega}^{\prime}\right\rangle$ are defined in a similar way.

\section{Existence of equilibria under Assumption 9}

As already observed, under Assumption 9,

$$
P=\left\{p \in \sum_{t=1}^{\infty} E_{i}\left|\sum_{t=1}^{\infty}\right| p_{t} \mid \cdot \omega_{t}<+\infty\right\}
$$

and $\omega \in \Lambda(P)$, the commodity-space constructed in Section 3 . So we can adapt to the case of incomplete and intransitive preferences the method of proof used by Aliprantis et al. [1] for infinite economies defined on a normal Riesz space.

First, we construct a sequence of finite economies consisting of a finite number of consumers. Applying a quasiequilibrium existence theorem (see Florenzano [14], Corollary 2 of the proposition 9), we remark that every finite economy has a quasiequilibrium with respect to the Riesz dual system $\left\langle A_{\omega}, A_{\dot{\omega}}^{\cdot}\right\rangle$. Then, by passing to limit, the economy $\mathscr{E}$ has a weak quasiequilibrium with respect to the same Riesz dual system.

In a second step, we show that if $(\bar{x}, \bar{p})$ is a weak quasiequilibrium and $\bar{q}$ is the order-continuous component of $\bar{p}$, then $(\bar{x}, \bar{q})$ is a quasiequilibrium. For such a result, the role of an assumption like 9 was stressed by Richard and Srivastava [19].

Finally, from the order-density of $A_{\omega}$ in $\Lambda(P)$ and the irreducibility of the economy, the quasiequilibrium with respect to the Riesz dual system $\left\langle A_{\omega}, A_{\omega}\right\rangle$ is shown to be actually an equilibrium with respect to the symmetric Riesz dual system $\langle\Lambda(P), P\rangle$.

Proposition 4.1. Under the assumptions 1-6 and 9, the economy 6 has a weak 
quasiequilibrium with respect to the Riesz dual system $\left\langle A_{\omega}, A_{\dot{\omega}}\right\rangle$.

Proof. Let $\mathscr{E}_{\mathbb{R}}$ be the economy constructed from $\mathscr{E}$ by considering the $m$ first consumers with for each $i, X^{i} \cap A_{\omega}$ as consumption set and the same preferences. As in Richard and Srivastava [19], the individual endowments are : $\omega^{1}(m)=\omega-\sum_{i=2}^{m} \omega^{i}$ and $\omega^{i}(m)=\omega^{i}$ for all $i: 2 \leq i \leq m$, so that the aggregate endowment of $\mathscr{E}_{k}$ is $\omega$. It could be verified that Proposition 9 in Florenzano [14] holds true if the assumption $\omega^{i} \in X^{i}$ is replaced by a survival assumption like $a^{i} \leq \omega^{i}$ for some $a^{i} \in X^{i}$. Then it is easily seen that the production economy, associated to $\mathscr{E}_{\mathbb{m}}$ by adding a producer of which the production set is equal to $Y=-A_{\omega}^{+}$, satisfies the other conditions of the corollary 2 of Proposition 9, when the two following topologies are considered on the commodity-space $A_{\omega}$ : the topology $\tau$, equal to the $\|.\|_{\omega^{-}}$ topology, and the topology $\sigma$ induced on $A_{\omega}$ by $\sigma(\Lambda(P), P)$. Hence $\mathscr{E}_{\mathbb{R}}$ has a free-disposal quasiequilibrium $\left(\bar{p}_{m}, \bar{x}_{m}\right)$ with $\bar{p}_{m} \in A_{\omega}^{\prime}$ and $\bar{p}_{m} \geq 0, \bar{p}_{m} \neq 0$. Since $\omega$ belongs to the $\|.\|_{\omega}$ interior of $A_{\omega}^{+}, \bar{p}_{\mathbb{R}}, \omega>0$ and we can assume $\bar{p}_{m} \cdot \omega=1$.

We finish the proof by passing to limit on the sequence of the free-disposal equilibria $\left(\bar{p}_{m}, \bar{x}_{m}\right)$. Let

$\Delta=\left\{p \in A_{\omega}^{\prime} \mid p \geq 0\right.$ and $\left.p . \omega=1\right\}$. From the Alaoglu-Bourbaki theorem, $\Delta$ is $\sigma\left(A_{\dot{\omega}}, A_{\omega}\right)$-compact. $\Delta \times[0, \omega]^{N}$ is then $\sigma\left(A_{\dot{\omega}}, A_{\omega}\right) \times(\sigma(\wedge(P), P))^{N}$-compact and we can assume that the sequence $\left(\bar{p}_{m}, \bar{x}_{m}^{-1}, \bar{x}_{m}^{2}, \ldots, \bar{x}_{m}^{-m}, 0, \ldots\right)_{m}$ of $\Delta \times[0, \omega]^{N}$ converges to $\left(\bar{p}, \bar{x}^{-1}, \bar{x}^{2}, \ldots\right) \in \Delta \times \prod_{i \in N}\left(x^{i} \cap A_{\omega}\right)$ endowed with the topology given above. Then, for $i \in N$, let $x^{i} \in X^{i} \cap A_{\omega}$ be such that $x^{i} \in P^{i}\left(\bar{x}^{i}\right)$. For sufficiently large $m, x^{i} \in P^{i}\left(\bar{x}_{m}^{i}\right)$ and $\bar{p}_{m} \cdot x^{i} \geq \bar{p}_{m} \cdot \omega^{i}(m)$. We distinguish two cases :

a) if $i=1, \bar{p}_{m} \cdot x^{1} \geq \bar{p}_{m} \cdot \omega^{1}(m)=\bar{p}_{m} \cdot\left(\omega-\sum_{i=2}^{m} \omega^{i}\right) \geq \bar{p}_{m} \cdot \omega^{1} \cdot$ By passing to limit, $\overline{\mathrm{p}} \cdot x^{1} \geq \bar{p} \cdot \omega^{1}$.

b) if $i \geq 2, \bar{p}_{m} \cdot x^{i} \geq \bar{p}_{m} \cdot \omega^{i}(m)=\bar{p}_{m} \cdot \omega^{i}$. As previously, $\bar{p} \cdot x^{i} \geq \bar{p} \cdot \omega^{i}$. Note that $\sum_{i=1}^{n} \bar{x}_{m}^{i} \leq \omega \quad \forall m \geq n$ and so $\sum_{i=1}^{n} \bar{x}^{i} \leq \omega$. Also $\bar{x}^{-i} \in X^{i}, \forall i \in N$. Let $x=\sup \left\{\sum_{i=1}^{n} x^{i} ; n=1,2, \ldots\right\} \quad\left(x\right.$ exists because $A_{\omega}$ is Dedekind- 
complete). At each period $t$, we can redistribute the surplus $\omega_{t}-x_{t}$ to a consumer $i$ such that $t \in T^{i}$. We get an allocation $\left(\bar{x}^{i}\right)_{i \in N}$ such that $\sum_{i=1}^{n} \bar{x}^{i} \uparrow \omega$. On the other hand, for all $t \in N$, we have in view of Assumption 5. $P^{i}\left(\bar{x}^{i}\right) \subset P^{i}\left(\bar{x}^{i}\right)$. It follows that $(\bar{p}, \overline{\bar{x}})$ is a weak quasiequilibrium of $\mathscr{E}$ with respect to the Riesz dual system $\left\langle A_{\omega}, A_{\dot{\omega}}\right\rangle$.

Proposition 4.2. Under the assumptions 1-6 and 9, the economy $\mathscr{E}$ has a quasiequilibrium with respect to the Riesz dual system $\left\langle A_{\omega}, A_{\omega}\right\rangle$ with a price in $\left(A_{\omega}\right)_{n}^{\sim}$.

Proof. Let $(\bar{p}, \overline{\bar{x}})$ be the weak quasiequilibrium given in the proof of Proposition 4.1 and let $\bar{p}=\bar{q}+r$ be the Riesz decomosition of $\bar{p}$, with $\bar{q} \in\left(A_{\omega}\right)_{n}^{\sim}(\bar{q}$ is the normal component of $\bar{p}), r \in\left(\left(A_{\omega}\right)_{n}^{\sim}\right)^{d}$, the disjoint complement of $\left(A_{\omega}\right)_{n}, \bar{q} \geq 0, r \geq 0$.

We first prove that $\bar{q}>0$. In view of Assumptions 4 and 9 , it is easily seen that there exists $j \in N$ such that $\overline{\mathrm{p}} \cdot \omega^{j}>\bar{p} \cdot \alpha^{j}$. Suppose that $\overline{\mathrm{q}}=0$. By Theorem 4.6 in Aliprantis and Burkinshaw [4], $0=\inf \left\{\sup \vec{p} \cdot x_{\alpha}, 0 \leq x_{\alpha} \uparrow x,\left(x_{\alpha}\right) \subset A_{\omega}\right\}$ for all $x \in A_{\omega}^{+}$. Let $\varepsilon=\bar{p} \cdot\left(\omega^{j}-a^{j}\right)=r \cdot\left(\omega^{j}-a^{j}\right)$ and $x^{j} \in X^{j} \cap A_{\omega}$ such that $x^{j} \in P^{j}\left(\overline{\bar{x}}^{j}\right)$. Then there exists a net $\left(x_{\alpha}\right) \subset A_{\omega}$ such that $0 \leq x_{\alpha} \uparrow x^{j}$ and $\sup r \cdot x_{\alpha}<\varepsilon$. This is impossible because, for sufficiently large $\alpha, x_{\alpha} \vee a^{j} \in P^{j}\left(\overline{\bar{x}}^{j}\right)$ and so $\varepsilon+r \cdot a^{j}>r \cdot\left(x_{\alpha} \vee a^{j}\right) \geq r \cdot \omega^{j}$.

Remark that because $\left(A_{\omega},\|\|,\right)$ is a Banach lattice, $A_{\dot{\omega}}=A_{\omega}^{\sim}$, the order dual of $A_{\omega}$. One deduces that $\bar{q} \in A_{\omega}^{\prime}$. From $\bar{q}>0$ and $\omega \in \operatorname{int}_{\|} \|_{\omega}\left(A_{\omega}^{+}\right)$, we get $\overline{\mathrm{q}} \cdot \omega>0$.

To see that $x^{i} \in P^{i}\left(\overline{\bar{x}}^{i}\right)$ implies $\bar{q} \cdot x^{i} \geq \bar{q} \cdot \omega^{i}$, assume that for some $j$. there exists $x^{j} \in P^{j}\left(\overline{\bar{x}}^{j}\right)$ with $\bar{q} \cdot x^{j}<\bar{q} \cdot \omega^{j}$. Let $\varepsilon=\bar{q} \cdot \omega^{j}-\bar{q} \cdot x^{j}$. From $\bar{q} \cdot x^{j}=\inf \left\{\sup (\bar{q}+r) \cdot x_{\alpha}, 0 \leq x_{\alpha} \uparrow x^{j},\left(x_{\alpha}\right) \subset A_{\omega}\right\}$, we deduce that $0=\inf \left\{\sup r \cdot x_{\alpha}, 0 \leq x_{\alpha} \uparrow x^{j},\left(x_{\alpha}\right) \subset A_{\omega}\right\}$. As previously, there exists a net $\left(x_{\alpha}\right) \subset A_{\omega}$ such that $0 \leq x_{\alpha} \uparrow x^{j}$ and $\sup r . x_{\alpha}<\varepsilon$. This is impossible since, for large enough $\alpha, x_{\alpha} \vee \alpha^{j} \in P^{j}\left(\overline{\bar{x}}^{j}\right)$, which implies $\overline{\mathrm{p}} \cdot \boldsymbol{\omega}^{j}=\overline{\mathrm{q}} \cdot \boldsymbol{\omega}^{j}+\boldsymbol{r} \cdot \boldsymbol{\omega}^{j} \leq \bar{q} \cdot\left(x_{\alpha} \vee \alpha^{j}\right)+\boldsymbol{r} \cdot\left(x_{\alpha} \vee \alpha^{j}\right)<$

$\bar{q} \cdot\left(x_{\alpha} \vee a^{j}\right)+\varepsilon+r \cdot a^{j}=\bar{q} \cdot\left(x_{\alpha} \vee a^{j}\right)+\bar{q} \cdot \omega^{j}-\bar{q} \cdot x^{j}+r \cdot a^{j} \leq$ $\overline{\mathrm{q}} \cdot \omega^{j}+r \cdot a^{j} \leq \overline{\mathrm{p}} \cdot \omega^{j}$.

It remains to prove that $\bar{q} \cdot \overline{\bar{x}}^{i}=\bar{q} \cdot \omega^{i}$ holds for all $t \in N$. That 
$\bar{q} \cdot \overline{\bar{x}}^{i} \geq \bar{q} \cdot \omega^{i}$ is easily deduced from the assumption 6 . Since $\bar{q}$ is ordercontinuous, we have $\sum_{i=1}^{n} \bar{q} \cdot \overline{\bar{x}}^{i} \uparrow \bar{q} \cdot \omega$; it easily follows that $\bar{q} \cdot \overline{\bar{x}}^{i}=\bar{q} \cdot \omega^{i}$ must hold for each $i$.

D

Proposition 4.3. Under the assumptions 1-9, the economy $\mathscr{E}$ has an equilibrium with respect to the symmetric Riesz dual system $\langle\Lambda(P), P\rangle$.

Proof. Recall that, under the assumptions 1,8 and $9, A_{\omega}$ is order-dense in $\Lambda(P)$ and $P=\left(A_{\omega}\right)_{n}^{\sim}$ (see Proposition 3.3). This proves that $\bar{q} \in P$. If $x^{i} \in X^{i} \cap \wedge(P)$ satisfies $x^{i} \in P^{i}\left(\overline{\bar{x}}^{i}\right)$, then there exists a net $\left(x^{i \nu}\right)_{\nu} \subset A_{\omega}$ such that $0 \leq x^{i \nu} \uparrow x^{i}$. For large enough $v, x^{i \nu} \vee a^{i} \in P^{i}\left(\overline{\bar{x}}^{i}\right)$ and thus $\bar{q} \cdot\left(x^{i v} \vee a^{i}\right) \geq \bar{q} \cdot \omega^{i}$. Passing to limit, we get $\bar{q} \cdot x^{i} \geq \bar{q} \cdot \omega^{i}$, which shows that $(\bar{q}, \overline{\bar{x}})$ is a quasiequilibrium with respect to $\langle\Lambda(P), P\rangle$. It follows now from the irreducibility of the economy (Assumption 7 ) that $(\bar{q}, \overline{\bar{x}})$ is actually an equilibrium.

It is worth noticing that the results of Section 4 could be proved under less restricting assumptions on the structure of consumption sets. Indeed, the conclusion of Proposition 4.1 still holds true if Assumption 3 is replaced by :

For each consumer $i, X^{i} \subset \prod_{t \in T^{i}} E_{t}^{+}$is convex, closed for the suitable product of the $\sigma\left(E_{t}, E_{t}^{\prime}\right)$-topologies, allows for free-disposal in $\prod_{t \in T^{i}} E_{t}^{+}$ and if, in Assumption 4, $a^{i}$ is some distinguished element of $x^{i}$. Then following Back's ideas [5], we can get the conclusion of Proposition 4.2 under the following additional condition :

For each $i$, each $x^{i} \in X^{i} \cap A_{\omega}$ and each $r \in\left(\left(A_{\omega}\right)_{n}^{\sim}\right)^{d}$, there exists in $X^{i} \cap A_{\omega}$ a net $\left(x^{i v}\right)$ order-convergent to $x^{i}$ such that $r \cdot x^{i v} \leq r \cdot a^{i}$.

This somewhat abstract condition is in particular satisfied, if each $E_{t}$ is finite dimensional, under an autarky condition like in Geanakoplos and Polemarchakis [16] :

For any $x^{i} \in X^{i} \cap \wedge(P)$, there exists $t_{0}$ such that for every $t \geq t_{0}$, $\hat{\mathrm{x}}^{i t} \in X^{i}$ with $\forall k \in T^{i}, \hat{\mathbf{x}}_{k}^{i t}=x_{k}^{i}$ if $k \leq t, \hat{\mathbf{x}}_{k}^{i t}=a_{k}^{i}$ if $k>t$. 
Under the same autarky condition, the conclusion of Proposition 4.3 is satisfied.

\section{Existence of equilibria in the overlapping generations model}

We now restrict our analysis to the overlapping generations model and thus replace Assumption 2 by Assumption $2^{\prime}$. We slightly modify Assumption 4 by assuming that $\lambda=\inf _{i} \lambda^{i}>0$; we strengthen too the continuity assumption on preferences and replace Assumptions 5 by :

$5^{1}$. For each $i, P^{i}: X^{i} \mapsto X^{i}$ has an open graph if $X^{i}$ is endowed with the suitable product of the $\sigma\left(E_{t}, E_{t}\right)$ - topologies. For every $x^{i} \in x^{i}$, $x^{i} \notin \operatorname{co} P^{i}\left(x^{i}\right)$ and $P^{i}\left(x^{i}\right) \subset P^{i}\left(y^{i}\right),\left(P^{i}\right)^{-1}\left(y^{i}\right) \subset\left(P^{i}\right)^{-1}\left(x^{i}\right)$ hold for all $x^{i}, y^{i} \in X^{i}$ such that $x^{i} \geq y^{i}$.

The approach followed in this section is borrowed from Burke [11] and adapted here to the case where at each period the commodity space may be infinite dimensional.

Let us consider an overlapping generations model satisfying the assumptions $1,2^{\prime}, 3,4,5^{\prime}, 6,7$ and 8 . By perturbing the initial endowments of the agents without changing the aggregate endowment of the economy, it is easy to associate to the initial model another one which satisfies Assumption 9 and thus, in application of the existence result of the previous section, has an equilibrium; this equilibrium can be seen as a tranfer equilibrium of the initial overlapping generations model.

By this way, we prove the existence of an infinite and relatively countably compact set of transfer equilibrium prices of our overlapping generations model. We then construct a net of transfer equilibria converging to a weak quasiequilibrium.

Finally, introducing Assumption 10 , we show that this weak quasiequilibrium is actually an equilibrium.

Recall that $P=\left\{p \in \prod_{t=1}^{\infty} E_{t}^{\prime}\left|\sum_{t=1}^{\infty}\right| p_{t} \mid \cdot \omega_{t}^{i}<+\infty, \forall i \in N\right\}$. It follows from Assumption 2' that for every $i \in N$, $X^{i} \subset \wedge(P)=\left\{x \in \prod_{t=1}^{\infty} E_{t}\left|\sum_{t=1}^{\infty}\right| p_{t}|.| x_{t} \mid<+\infty, \forall p \in P\right\}$.

A transfer equilibrium of the overlapping generations model consists of 
a price $\overline{\mathrm{p}} \in P$ and an attainable allocation $\overline{\mathrm{x}} \in \prod_{i \in N} x^{i}$ such that $\overline{\mathrm{p}} \cdot \bar{x}^{i}>0$ for each $t \in N$ and $x^{i} \in P^{i}\left(\bar{x}^{-i}\right)$ implies $\bar{p} \cdot x^{i}>\bar{p} \cdot \bar{x}^{i}$.

It is worth remarking that the existence of transfer equilibria follows directly from Proposition 4.3. Moreover we have the following :

Proposition 5.1. Let $k_{0}$ be the smallest integer $k$ satisfying $k>\frac{2}{\lambda}$. Under Assumptions $1,2^{\prime}, 3,4,5^{\prime}, 6,7$ and $8, \mathscr{E}$ has at least countably many transfer equilibria $(\bar{p}, \bar{x})$ satisfying

$\sum_{t=1}^{\infty} \bar{p}_{t} \cdot \omega_{t}<+\infty, \bar{p} \cdot \bar{x}^{-1}=1$

and for every $i, \overline{\mathrm{p}} \cdot \overline{\mathrm{x}}^{i} \geq\left(1-\frac{2}{k_{0}}\right) \bar{p} \cdot \omega^{i}>0$.

Proof. Choose $\varepsilon=\sum_{i} \varepsilon^{i}$ such that for every $i, 0 \leq \varepsilon^{i} \leq \omega^{i}$ and for every $t$, $\varepsilon_{t}$ is a strictly positive element of $E_{t}$. For $k \geq k_{0}$, form the $k$-perturbed economy $\delta^{k}$ by redistributing endowments from $\left(\omega^{i}\right)$ to $\left(\omega^{i k}\right)$, where

$$
\begin{aligned}
& \omega^{i k}=\left(1-\frac{1}{k}\right) \omega^{i}+\frac{1}{k}\left(\frac{\varepsilon}{2^{i}}-\varepsilon^{i}\right) \text { for } i \neq k \\
& \omega^{k k}=\left(1-\frac{1}{k}\right) \omega^{k}+\frac{1}{k}\left(\frac{\varepsilon}{2^{k}}-\varepsilon^{k}\right)+\frac{1}{k} \omega .
\end{aligned}
$$

We remark that for every $i, \omega^{i k} \geq\left(1-\frac{2}{k}\right) \omega^{i}+\frac{1}{k} \frac{\varepsilon}{2^{i}}$ with $\omega^{k k} \geq \frac{1}{k} \omega$ and that $\sum_{i \in N} \omega^{i k}=\sum_{i \in N} \omega^{i}=\omega$. Clearly, Proposition 4.3 establishes the existence of a quasiequilibrium $\left(\bar{x}^{k}, \bar{p}^{k}\right)$ of $\sigma^{k}$ such that for every $i$, $\overline{\mathrm{p}}^{-k} \cdot \bar{x}^{i k}=\bar{p}_{\infty}^{-k} \cdot \omega^{i k}$, with a price $\bar{p}^{-k}>0$ in

$Q=\left\{p \in \prod_{t=1}^{\infty} E_{t}\left|\sum_{t=1}^{\infty}\right| p_{t} \mid \cdot \omega_{t}<+\infty\right\}$. As for every $i, \bar{p}^{-k} \cdot \omega^{i k}>\bar{p}^{-k} \cdot a^{i}$. this quasiequilibrium is an equilibrium of $\mathscr{6}^{k}$ hence a transfer
equilibrium of $\mathscr{E}$. Note that for every $i, \mathbf{p}^{-k} \cdot \bar{x}^{-i k} \geq \mathbf{p}^{-k} \cdot\left(1-\frac{2}{k}\right) \omega^{i} \geq$ $\left(1-\frac{2}{k_{0}}\right) p^{-k} \cdot \omega^{i}$. We can normalize the vector price $\bar{p}^{-k}$ so as to get $\bar{p}^{-k} \cdot \bar{x}^{-1 k}=1$. Finally $\bar{p}^{-k} \cdot \omega>0$ and, since $\bar{p}^{-k} \in Q, \bar{p}^{-k} \cdot \omega^{i}>0$ for some $t$. That $\bar{p}^{k} \cdot \omega^{i}>0$ for every $i$ follows from Assumption 7 .'

The next result in an analogue of Lemma 3 in Wilson [21], Lemma 8.5 in 


\section{Aliprantis et al [1].}

Lemma 5.2. Under the assumptions $1,2^{\prime}, 3,4,5^{\prime}, 6,7$ and 8 , if $\left(x^{-n}, \bar{p}^{-n}\right)$ is any sequence of transfer equilibria satisfying the properties required in Proposition 5.1, then for any couple $(i, j)$ there exists $K_{i j}$ such that for $n$ large enough

$$
0<\bar{p}^{-n} \cdot \bar{x}^{-i n}<k_{i j} \bar{p}^{-n} \cdot \bar{x}^{-j n}
$$

Proof. The proof is conducted by contraposition. Assume that $\left(i_{0}, j_{0}\right)$ is a couple of agents such that $\lim _{n \rightarrow \infty} \frac{\inf ^{-n} \cdot \bar{x}^{-j} o^{n}}{\bar{p}^{n} \cdot \bar{x}^{-i} o^{n}}=0$. Then

$I=\left\{i \in N \mid \lim _{n \rightarrow \infty} \frac{\bar{p}^{n} \cdot \bar{x}^{-i n}}{\bar{p}^{n} \cdot \bar{x}^{-i} 0^{n}} \neq 0\right\}$ is a non-empty and proper subset of $N$.

By passing to a subsequence (built by diagonal process), we can assume that $\lim _{n \rightarrow \infty} \frac{\bar{p}^{-n} \cdot \bar{x}^{-i n}}{\bar{p}^{-n} \cdot \bar{x}^{-i} o^{n}}>0 \forall i \in I \quad$ and $\lim _{n \rightarrow \infty} \frac{\bar{p}^{-n} \cdot \bar{x}^{-j n} \cdot \bar{x}^{-i o^{n}}}{p^{-j n}}=0 \quad \forall j \notin I$.

In view of $\bar{x}^{-i n} \in \prod_{t \in T^{i}}\left[0, \omega_{t}\right]$ and the $\sigma\left(E_{t}, E_{t}\right)$ - compactness of the order intervals, we see that the sequence $\left(\bar{x}^{-n}\right)$ has a subnet $\left(\bar{x}^{-\alpha}\right)$ converging to $\bar{x}$ when $X=\prod_{i \in N} x^{i}$ is endowed with the product of the suitable product of the $\sigma\left(E_{t}, E_{t}^{\prime}\right)$-topologies on each $X^{i}$. It follows from $2^{\prime}$ that $\bar{x}$ is an attainable allocation. From 7 , we deduce that there exist $i \in I, z^{i} \in \prod_{t \in T^{i}} E_{t}^{+}$and a finite subset $F$ of $N \backslash I$ such that $z^{i} \leq \sum_{j \in F} \omega^{j}$ and $\bar{x}^{i}+z^{i} \in P^{i}\left(\bar{x}^{i}\right)$. Using $5^{\prime}$, we see that there exist some $\delta, 0<\delta<1$ and some $\alpha_{0}$ such that $(1-\delta) \alpha^{i}+\delta\left(\bar{x}^{-i \alpha}+z^{i}\right) \in P^{i}\left(\bar{x}^{i \alpha}\right)$ for all $\alpha \geq \alpha_{0}$. So we can built a stricly increasing sequence $n_{k}$ of integers satisfying $(1-\delta) a^{i}+\delta\left(\bar{x}^{i n_{k}}+z^{i}\right) \in P^{i}\left(\bar{x}^{i n_{k}}\right)$. From this we deduce : $\overline{\mathrm{p}}^{-n_{k}} \cdot \overline{\mathrm{x}}^{-i n_{k}}<\bar{p}^{-n_{k}} \cdot a^{i}+\frac{\delta}{1-\delta} \bar{p}^{-n_{k}} \cdot \mathrm{z}^{i} \leq \frac{1-\lambda}{1-\frac{2}{k_{0}}} \bar{p}^{-n_{k}} \cdot \bar{x}^{i n_{k}}+\frac{\delta}{1-\delta} \bar{p}^{-n_{k}} \cdot \sum_{j \in F} \omega^{j}$ 
$\leq \frac{1-\lambda}{1-\frac{2}{k_{0}}} \bar{p}^{-n_{k}} \cdot \bar{x}^{-i n_{k}}+\left(\frac{\delta}{1-\delta}\right)\left(\frac{1}{1-\frac{2}{k_{0}}}\right) \sum_{j \in F} \bar{p}^{-n_{k}} \cdot \bar{x}^{-j n_{k}}$.

Then $\left(\lambda-\frac{2}{k_{0}}\right) \bar{p}^{-n_{k}} \cdot \bar{x}^{-i n_{k}} \leq \frac{\delta}{1-\delta} \sum_{j \in F} \bar{p}^{-n_{k}} \cdot \bar{x}^{-j n_{k}}$, which contradicts the assumption made on $F$ and $i$.

For each $t$, let $E_{t}^{*}$ denote the norm dual of $E_{t}$ for the Riesz norm $\left\|x_{t}\right\|_{\infty}=$ inf $\left\{\lambda>0|| x_{t} \mid \leq \lambda \omega_{t}\right\}$. Recall that $E_{t}^{*}=E_{t}^{n}$, the order-bounded dual of $E_{t}$, and that $E_{t}^{\prime}=\left(E_{t}\right)_{n}^{\sim}$.

Lemma 5.3. Under the same assumptions and conditions, $\left(\bar{p}^{-n}, \bar{x}^{-n}\right)$ has a subnet converging to $(\bar{p}, \bar{x}) \in \prod_{t=1} E_{t} ; \times \prod_{i \in N} x^{i}$ for $\prod_{t=1}^{\infty} \sigma\left(E_{t}^{*}, E_{t}\right) \times \prod_{i \in N}\left(\prod_{t \in T^{i}} \sigma\left(E_{t}, E_{t}^{\prime}\right)\right)$, such that for every $i, x^{i} \in P^{i}\left(\bar{x}^{i}\right)$ implies $\bar{p} \cdot x^{i} \geq \lim _{n} \bar{p}^{n} \cdot \bar{x}^{-i n}$.

Proof. Note that in view of Lemma 5.2, we can assume w.1.o.g. that the sequence $\bar{p}^{-n} \cdot \bar{x}^{i n}$ converges and that $i$ im $\vec{p}^{n} \cdot \bar{x}^{i n}>0$. It follows also from Lemma 5.2 and Assumption 2' that for ' each $t$, there exists $k_{t}$ such that $\bar{p}_{t}^{n} \cdot \omega_{t}<k_{t}$ if $n$ is large enough. From this and the weak compactness of the order intervals, we deduce that $\left(\bar{p}^{n}, \bar{x}^{n}\right)$ has a subnet $\left(\bar{p}^{\alpha}, \bar{x}^{-\alpha}\right)$ converging to $(\bar{p}, \bar{x}) \in \prod_{t=1}^{\infty} E_{t}^{*} \times \prod_{i \in N} x^{i}$ for the topology $\prod_{t=1}^{\infty} \sigma\left(E_{t}^{*}, E_{t}\right) \times$

$\prod\left(\prod \sigma\left(E_{t}, E_{t}^{\prime}\right)\right)$. As previously, $\bar{x}$ is an attainable allocation.

$i \in N \quad t \in T^{i}$

For every $i$, if $x^{i} \in P^{i}\left(\bar{x}^{i}\right)$ then for $\alpha$ large enough, $x^{i} \in P^{i}\left(\bar{x}^{i \alpha}\right)$ and thus $\bar{p}^{-\alpha} \cdot x^{i}>\bar{p}^{-\alpha} \cdot \bar{x}^{i \alpha}$. By passing to limit, we get

$$
\overline{\mathrm{p}} \cdot x^{i} \geq \lim _{\alpha} \bar{p}^{-\alpha} \cdot \bar{x}^{-i \alpha}=\lim _{n} \bar{p}^{-n} \cdot \bar{x}^{-i n}
$$

Note that $\overline{\mathrm{p}} \neq 0$.

Moreover for every $t$, let $y_{t}^{\beta}+0$ hold in $E_{t}$. By scaling appropriately, we can suppose that $y_{t}^{\beta} \leq \omega_{t}-\alpha_{t}$. If $I_{t}$ denotes the $\operatorname{set}\left\{i \in N \quad t \in T^{i}\right\}$, it follows from $2^{\prime}$ and the decomposition property of vector lattices that $y_{t}^{\beta}=\sum_{i \in I_{t}} y_{t}^{i \alpha \beta}$ with $0 \leq y_{t}^{i \alpha \beta} \leq \bar{x}_{t}^{i \alpha}-\alpha_{t}^{i}$. From $0 \leq y_{t}^{i \alpha \beta} \leq y_{t}^{\beta} \downarrow 0$ and the fact 
that each dual system $\left\langle E_{t}, E_{t}\right\rangle$ is symmetric, it follows that $y_{t}^{i \alpha \beta} \rightarrow 0$ for $\sigma\left(E_{t}, E_{t}\right)$. Now fix $\varepsilon>0$ and note that it follows from Assumption 6 that for each $i, \bar{x}^{-i}+z^{i} \in P^{i}\left(\bar{x}^{i}\right)$ for some $z^{i} \in \prod E_{t}^{+}, z^{i} \leq \varepsilon \omega$. Then there exist $\alpha_{0}$ and $\beta_{0}$ such that

$$
\bar{x}^{-i \alpha}+z^{i}-\left(0, \ldots, y_{t}^{i \alpha \beta}, 0, \ldots\right) \in P^{i}\left(\bar{x}^{i \alpha}\right)
$$

hold for all $\alpha \geq \alpha_{0}, \beta \geq \beta_{0}$ and each $i \in I_{t}$. From this we infer that $\overline{\mathrm{p}}_{t}^{-\alpha} \cdot y_{t}^{i \alpha \beta} \leq \varepsilon \bar{p}^{-\alpha} \cdot v^{i}$ where $v^{i}$ is the projection of $\omega$ onto $\prod_{t \in T^{i}} E_{t}$, and thus that $\overline{\mathrm{p}}_{t}^{-\alpha} \cdot y_{t}^{i \beta} \leq \varepsilon \bar{p}^{-\alpha} \cdot \sum_{i \in I_{t}} v^{i}$. Taking limits with respect to $\alpha$, we get $: \overline{\mathrm{p}}_{t} \cdot y_{t}^{\mathcal{\beta}} \leq \varepsilon \bar{p} \cdot \sum_{i \in I_{t}} v^{i}$ for all $\beta \geq \beta_{0}$. Hence $\overline{\mathrm{p}}_{t} \cdot y_{t}^{\beta} \mapsto 0$. $\overline{\mathrm{p}}_{t}$ is order-continuous and thus belongs to $E_{\dot{t}}^{\prime}$.

We can now prove the main existence result of this section .

Proposition 5.4. Under the assumptions 1,2', 3, 4, 5', 6, 7 and 8, the overlapping generations model $\mathscr{E}$ has a weak quasiequilibrium $(\bar{x}, \bar{p})$, with respect to the symmetric Riesz dual system $\langle\Lambda(P), P\rangle$, such that for each $i \in N:$

$$
\begin{aligned}
& \bar{p} \cdot \bar{x}^{i} \geq \bar{p} \cdot \omega^{i} \\
& \bar{p} \cdot \bar{x}^{i}=\bar{p} \cdot \omega^{i} \text {, if } \omega^{i} \text { has only finitely many non-zero coordinates. }
\end{aligned}
$$

Proof. Let $Q_{\lambda}$ denote the collection of the price systems corresponding to transfer equilibria satisfying the properties required in Proposition 5.1. We first observe that it follows from Lemma 5.3 that $Q_{\lambda}$ is $\prod_{t=1}^{\infty} \sigma\left(E_{t}^{*}, E_{t}\right)-$ relatively countably compact and thus $\prod_{t=1}^{\infty} \sigma\left(E_{t}^{*}, E_{t}\right)$-bounded (see for example Köthe [17], p. 310). So we can choose $\varepsilon=\left(\varepsilon_{t}\right)$ such that $\varepsilon=\sum_{i \in N} \varepsilon^{i}$, $0 \leq \varepsilon^{i} \leq \omega^{i}$ for every $t, \varepsilon_{t}$ is an order-unit of $E_{t}$ for every $t$ and $p$. $\varepsilon \leq 1$ for every $p$ in $Q_{\lambda}$. Let $\left(\mathscr{C}^{\star}\right)$ be the sequence of the $k$-perturbed 
economies defined in the proof of Proposition 5.1 and $\left(\bar{x}^{k}, \bar{p}^{-k}\right)$ the corresponding sequence of transfer equilibria of $\mathscr{E}$. As for each $i$,

$$
\bar{p}^{-k} \cdot \bar{x}^{-i k}=\bar{p}^{-k} \cdot \omega^{i k}=\left(1-\frac{1}{k}\right) \bar{p}^{-k} \cdot \omega^{i}+\frac{1}{k} \bar{p}^{-k} \cdot\left(\frac{\varepsilon}{2^{i}}-\varepsilon^{i}\right) \text {. }
$$

we get by passing to limit in a suitable subnet converging to $(\bar{p}, \bar{x})$ : $\lim _{k} \bar{p}^{-k} \cdot \bar{x}^{-i k}=\lim _{k} \bar{p}^{k} \cdot \omega^{i}$, which shows that $\lim _{k} \bar{p}^{-k} \cdot \omega^{i}$ exists, and, in application of Lemma 5.3. $x^{i} \in P^{i}\left(\bar{x}^{i}\right)$ implies $\overline{\mathrm{p}} \cdot x^{i} \geq \lim _{k} \bar{p}^{k} \cdot \omega^{i}$.

It is easily deduced from Assumption 6 that for every $t$ and for each $i \in I_{t}, \quad \bar{p}_{t} \cdot \bar{x}_{t}^{i} \geq \lim _{k} \bar{p}_{t}^{k} \cdot \bar{x}_{t}^{i k}$. As $\quad \sum_{i \in I_{t}} \bar{p}_{t} \cdot \bar{x}_{t}^{i}=\bar{p}_{t} \cdot \sum_{i \in I_{t}} \bar{x}_{t}^{i}=$ $\lim _{k} \bar{p}_{t}^{k} \cdot \sum_{i \in I_{t}} \bar{x}_{t}^{-i}=\lim _{k} \bar{p}_{t}^{k} \cdot \sum_{i \in I_{t}} \bar{x}_{t}^{i k}=\sum_{i \in I_{t}} \lim _{k} \bar{p}_{t}^{k} \cdot \bar{x}_{t}^{i k}$, we then have for every $t$ and for each $i \in I_{t}, \bar{p}_{t} \cdot \bar{x}_{t}^{i}=\lim _{k} \bar{p}_{t}^{k} \cdot \bar{x}_{t}^{-i k}$. Finally, for every $i, \overline{\mathrm{p}} \cdot \bar{x}^{-i}=\lim _{k} \bar{p}^{-k} \cdot \bar{x}^{-i k}=\lim _{k} \bar{p}^{-k} \cdot \omega^{i}$.

Obviously, $\underset{k}{\lim } \bar{p}^{-k} \cdot \omega^{i}=\bar{p} \cdot \omega^{i}$ if $\omega^{i}$ has only finitely many non-zero coordinates. If not, from $\forall k, \forall T, \sum_{t=1}^{T} \bar{p}_{t}^{k} \cdot \omega_{t}^{i} \leq \bar{p}^{-k} \cdot \omega^{i}$ we get $\forall T$, $\sum_{t=1}^{T} \bar{p}_{t} \cdot \omega_{t}^{i} \leq \lim _{k} \bar{p}^{-k} \cdot \omega^{i}$ and, thus, $\bar{p} \in P$ and $\bar{p} \cdot \omega^{i} \leq \lim _{k} \bar{p}^{k} \cdot \omega^{i}$. As $\bar{x}$ is attainable and $\bar{p} \neq 0,(\bar{x}, \bar{p})$ is a weak quasiequilibrium with respect to the Riesz dual system $\langle\wedge(P), P\rangle$.

Remark. As for every $i, \lim _{k} \bar{p}^{k} \cdot \bar{x}^{i k}>0,(\bar{x}, \bar{p})$ is what is called a compensated equilibrium in Geanakoplos and Polemarchakis [16]. Thus Proposition 5.4 has a first obvious corollary :

Corollary 5.5. Under the assumptions of Proposition 5.4 and Assumption 10, if $\omega^{\prime}=0$, the overlapping generations model has an equilibrium with respect to the symmetric Riesz dual system $\langle\Lambda(P), P\rangle$.

If $\omega^{\prime}>0$, under Assumption 10, $P=\left\{p \in \prod_{t=1}^{\infty} E_{t}\left|\sum_{t=1}^{\infty}\right| p_{t} \mid \cdot \omega_{t}^{t}<+\infty\right\}$. 
Let, as in Assumption 10, $I_{0}$ finite and $\theta>0$ be such that $\sum_{i \in I_{0}} \omega^{\prime i} \geq \theta \omega^{\prime}$. It can easily be verified that the proofs and the results of the lemmas 5.2 and 5.3 and Proposition 5.4 are not altered if we change the price normalization used in the statement of Proposition 5.1 from $\bar{p} \cdot \bar{x}^{-1}=1$ to $\bar{p} \cdot \sum_{i \in I_{0}} \bar{x}^{i}=1$. Let then $\left(\mathscr{C}^{k}\right)$ be the sequence of the $k$-perturbed economies and $(\bar{x}, \bar{p})$ the corresponding compensated equilibrium of $\mathscr{E}$. It follows from the price normalization that for every $k, \bar{p}^{k} \cdot \omega^{\prime} \leq \frac{1}{\theta} \sum_{i \in I_{0}} \bar{p}^{k} \cdot \omega^{i} \leq$ $\frac{1}{\theta} \sum_{i \in I_{0}} \bar{p}^{k} \cdot \omega^{i} \leq \frac{1}{(1-\lambda) \theta} \sum_{i \in I_{0}} \bar{p}^{k} \cdot \bar{x}^{-i k}=\frac{1}{(1-\lambda) \theta}$. Clearly, since for every $i$, $\overline{\mathrm{p}} \cdot \bar{x}^{-i}=\lim _{k} \bar{p}^{k} \cdot \omega^{+i}+\bar{p} \cdot \omega^{\prime i}$

$(\bar{x}, \bar{p})$ is either an equilibrium of $\mathscr{E}$ or only a Samuelson-type monetary equilibrium, according as $\bar{p} \cdot \omega^{\prime}=\lim _{k} \bar{p}^{-k} \cdot \omega^{\prime}$ or $\bar{p} \cdot \omega^{\prime}<\lim _{k} \bar{p}^{-k} \cdot \omega^{\prime}$. Examples of economies with only Samuelson-type monetary equilibria show that the above equality cannot be guaranteed without some additional assumption. We propound now such an assumption which insures informally that $\omega^{\prime}$ is a good substitute for every agent and which links the marginal rate of substitution for each agent with the development of $\left(\omega_{t}^{\prime}\right)$.

For every $i$, let $v^{\prime i}$ be the projection of $\omega^{\prime}$ onto $\prod E_{t}$. $t \in T^{i}$

11. For every $t$, there exists some neighborhood $V_{t}$ of 0 in $E_{t}$ such that :

- For every $x \in \hat{x}$, for each $t$, for every $\lambda>0$ and for every $u \in \prod v_{t}$, $x^{i}+\lambda v^{\prime i}+\lambda u \in X^{i}$ implies $x^{i}+\lambda v^{\prime i}+\lambda u \in P^{i}\left(x^{i}\right)$

- For every $t$, $q_{t}\left(\omega_{t}^{i}\right) \leq \frac{1}{2^{t} n_{t}}$ ( where $q_{t}$ is the gauge of $v_{t}$ and $n_{t}$ the number of agents alive at $t$ ).

Corollary 5.6. Under the assumptions of Proposition 5.4, Assumption 10 and Assumption 11 if $\omega^{\prime}>0$, the overlapping generations model has an equilibrium with respect to the symmetric Riesz dual system $\langle\Lambda(P), P\rangle$. 
Proof. Let $k \geq k_{0}$ be given. For every $t$, consider $z_{t}$ such that $0 \leq z_{t} \leq \lambda \omega_{t} \leq \omega_{t}-a_{t}$. In view of the decomposition property of vector lattices, $z_{t}=\sum_{t \in I(t)} z_{t}^{i}$ with $0 \leq z_{t}^{i} \leq \bar{x}_{t}^{-i k}-a_{t}^{i}$. For each $t \in I(t)$, if $\alpha>0$ and $\hat{z}^{i}=\left\{\begin{array}{c}z_{t}^{i} \text { at period } t \\ 0 \text { elsewhere }\end{array}\right.$ then $\overline{\mathbf{x}}^{i k}+\left(q_{t}\left(z_{t}^{i}\right)+\alpha\right) v^{\prime i}-\hat{z}^{i} \in P^{i}\left(\bar{x}^{i k}\right)$. Hence, $\bar{p}_{t}^{k} \cdot z_{t}^{i}<\left(q_{t}\left(z_{t}^{i}\right)+\alpha\right) \bar{p}^{-k} \cdot v^{\prime^{i}} \leq \frac{1}{(1-\lambda) \theta}\left(q_{t}\left(z_{t}^{i}\right)+\alpha\right)$. Summing over $i \in I(t)$. we get $\bar{p}_{t}^{k} \cdot z_{t}<\frac{1}{(1-\lambda) \theta} n_{t}\left(q_{t}\left(z_{t}\right)+\alpha\right)$ and since $\alpha$ is arbitrary, $\bar{p}_{t}^{-k} \cdot z_{t} \leq \frac{1}{(1-\lambda) \theta} n_{t} q_{t}\left(z_{t}\right)$.

From the second part of Assumption 11, we deduce that for every $k$, for every $t \quad: \quad \bar{p}_{t}^{-k} \cdot \lambda \omega_{t} \leq \frac{\lambda}{(1-\lambda) \theta} \frac{1}{2^{t}}$. It is now obvious that $\lim _{k} \bar{p}^{-k} \cdot \omega^{\prime} \leq \bar{p} \cdot \omega^{\prime}$ and thus that $\lim _{k} \bar{p}^{-k} \cdot \omega^{\prime}=\bar{p} \cdot \omega^{\prime}$.

As it is well known, the equilibrium whose existence is proved by Corollary 5.5 or Corollary 5.6 may fail to be Pareto optimal.

Let $\varepsilon=\left(\varepsilon_{t}\right)$ be as in the proof of Proposition 5.4. Let us say that $a$ finite set of non-negligible agents exists everywhere, if and only if for any attainable allocation, $x$, there exists a finite set of agents $I_{x} \subset N$, a scalar $\alpha>0$ and an allocation $x^{\prime}$ such that

$\sum_{i \in N} x^{\prime i}=\propto \sum_{i \in I_{x}} \omega^{i}+\varepsilon$

and, for each $i$.

$x^{1^{i}} \in \overline{P^{i}\left(x^{i}\right)}$ (the closure of $P^{i}\left(x^{i}\right)$ for the product of the $\sigma\left(E_{t}, E_{t}\right)$ topologies).

The definition, evidently, generalizes the assumption 9.

The following result improves Theorem 2 in Geanakoplos and Polemarchakis (1990) and strengthens for overlapping generations models the existence result of Proposition 4.3 .

Corollary 5.7. Under the assumptions of Proposition 5.4, if a finite set of non-negligible agents exists everywhere, the overlapping generations model 
has an equilibrium $(\bar{x}, \bar{p})$ such that $\sum_{t=1}^{\infty} \bar{p}_{t} \cdot \omega_{t}<+\infty$.

Proof. Let $(\bar{x}, \bar{p})$ be the compensated equilibrium got from Proposition 5.4 . Let $I_{\bar{x}}, x^{\prime}$ and $\alpha$ be as in the definition of a finite set of non-negligible agents. Note that $\bar{p} \cdot \varepsilon=\sum_{t=1}^{\infty} \bar{p}_{t} \cdot \varepsilon_{t} \leq 1$. For each $i, \bar{p} \cdot x^{\prime i} \geq \bar{p} \cdot \omega^{i}$, and hence $\bar{p} \cdot \omega \leq 1+\alpha \sum_{i \in I} \bar{p} \cdot \omega^{i}<+\infty$. Since $\bar{p} \cdot \bar{x}^{i} \geq \bar{p} \cdot \omega^{i}$ while $\sum_{i \in N} \bar{x}^{i}=\omega$, $\bar{p} \cdot \bar{x}^{i}=\bar{p} \cdot \omega^{i}$ for $i \in N$.

口

Remark. At the cost of a slight strengthening of the desirability assumption on $\omega$, the previous results could easily be extended to a model which allows at each period for a finite number of both finite-lived and infinite-lived agents, so as to get an existence result similar to that of Wilson [21] or Burke [11]. But it should be understood that the equilibrium concept used by Wilson and Burke does not coincide in this case with the definition given in this paper of an equilibrium with respect to the Riesz dual system $\langle\Lambda(P), P\rangle$.

\section{The overlapping generations model with proper preferences}

Recall that for each $i$, the preference $P^{i}$ is said to be uniformly proper if there exist some $v^{i} \neq 0$ in $\prod E_{t}^{+}$and some neighborhood $v^{i}$ of zero in $t \in T^{i}$

$X^{i}$ for the suitable product of the topologies given on $E_{t}$ such that for every $x \in X$, for every $\lambda>0$, for every $u \in V^{i}, x^{i}+\lambda v^{i}+\lambda u \in X^{i}$ implies $x^{i}+\lambda v^{i}+\lambda u \in P^{i}\left(x^{i}\right)$.

If each preference $P^{i}$ is uniformly proper, we can weaken Assumption 8 to :

$8^{\prime}$. For every $t, \omega_{t}$ is a strictly positive element of $E_{t}$

and prove the following result which extends the previous existence results to overlapping generations models with a commodity-price duality represented at each period by $\left\langle L_{p}(\mu), L_{q}(\mu)\right\rangle \quad(1<p<\infty, 1<q<\infty$, 
$\left.\frac{1}{p}+\frac{1}{q}=1\right)$

Proposition 6.1. Under the assumptions 1, 2', 3, 4, 5', 6, 7, 8', 10 and, in addition, 11 if $\omega^{\prime}>0$, if each preference $P^{i}$ is uniformly proper, then the overlapping generations model has an equilibrium.

Proof. Let for each $t, A_{\omega_{t}}$ be the ideal generated by $\omega_{t}$ in $E_{t}$. It follows from Corollaries 5.5 and 5.6 that the overlapping generations model has an equilibrium $(\bar{x}, \bar{p})$ relative to the symmetric Riesz dual system $\left\langle\Phi, \prod_{t=1}^{\infty}\left(A_{\omega_{t}}\right)_{n}\right\rangle$ where $\Phi$ denotes the subset of $\prod_{t=1}^{\infty} A_{\omega_{t}}$ of all sequences with only finitely many non-zero components.

In order to prove that each $\overline{\mathrm{p}}_{t}$ is continuous on $A_{\omega_{t}}$ endowed with the topology of $E_{t}$, we adapt arguments from Yannelis and Zame [22], Aliprantis et ar. [1].

We first remark that in view of $2^{\prime}, 5^{\prime}$ and $8^{\prime}$, there is no loss of generality to assume that for every $i$, the uniform properness vector is $v^{i}=\left(v_{t}^{i}\right)$ with $v_{t}^{i}=\omega_{t}$ if $t \in T^{i}$ and $v_{t}^{i}=0$ if $t \in T^{i}$. We can also assume that for each $i$, the uniform properness neighborhood is $V^{i}=\prod_{t \in T^{i}} v_{t}^{i}$. Let $t$ be some fixed period, $I(t)$ the set of all agents alive at period $t$. We define $V_{t}=\bigcap_{i \in I(t)} V_{t}^{i}$ and on $E_{t}$, $P_{t}$ the gauge of $V_{t}$. As in the proof of Corollary 5.6, let now $z_{t}$ be such that $0 \leq z_{t} \leq \lambda \omega_{t} \leq \omega_{t}-a_{t}$ and fix $\alpha>0$. In view of the decomposition property of vector lattices, $z_{t}=\sum_{i \in I(t)} z_{t}^{i}$ with $0 \leq z_{t}^{i} \leq \bar{x}_{t}^{i}-a_{t}^{i}$. For each $i \in I(t)$, in view of the uniform properness of $P^{i}$, if $\hat{z}^{i}=\left\{\begin{array}{l}z_{t}^{i} \text { at period } t \\ 0 \text { elsewhere }\end{array}\right.$ then $\bar{x}^{i}+\left(q_{t}\left(z_{t}^{i}\right)+\alpha\right) v^{i}-\hat{z}^{i} \in P^{i}\left(\bar{x}^{i}\right)$. Thus $\overline{\mathrm{p}} \cdot \bar{x}^{-i}+\left(q_{t}\left(z_{t}^{i}\right)+\alpha\right) \bar{p} \cdot v^{i}-\bar{p} \cdot \hat{z}^{i}>\overline{\mathrm{p}} \cdot \bar{x}^{-i}$ and $\bar{p}_{t} \cdot z_{t}^{i}<\left(q_{t}\left(z_{t}^{i}\right)+\alpha\right) \bar{p} \cdot v^{i}$. Since $q_{t}$ is a monotone seminorm, we deduce $\bar{p}_{t} \cdot z_{t}^{i}<\left(q_{t}\left(z_{t}\right)+\alpha\right) \bar{p} \cdot v^{i}$ and $\bar{p}_{t} \cdot z_{t}<\left(q_{t}\left(z_{t}\right)+\alpha\right) \sum_{i \in I(t)} \bar{p} \cdot v^{i}$. Since the previous assertion is satisfied for every $\alpha>0$, 
$\bar{p}_{t} \cdot z_{t} \leq q_{t}\left(z_{t}\right) \sum_{i \in I(t)} \bar{p} \cdot v^{i}$. This shows that $\bar{p}_{t}$ is continuous on $A_{\omega_{t}}$ for the topology given on $E_{t}$.

Since $A_{\omega_{t}}$ is dense in $E_{t}, \bar{p}_{t}$ has a unique continuous extension $\overline{\bar{p}}_{t}$ on $E_{t}$. A last density argument shows that $(\bar{x}, \overline{\bar{p}})$ is a quasiequilibrium and, since $\overline{\bar{p}} \cdot \omega^{i}=\bar{p} \cdot \omega^{i}>0$, an equilibrium.

\section{Références}

1. Aliprantis, C.D.. Brown, D.J., Burkinshaw, o.: Existence ln exchange economles with countable number of agents, J. Math. Anal. App1. 142, $250-299(1989)$

2. Aliprantis, C.D., Brown D.J., Burkinshaw, O.: Existence and optimality of competitive equilibria. Berlin, Heidelberg. New York: Springer 1989

3. Aliprantis, C.D.. Burkinshaw, O.: Locally Solid Rlesz Spaces. New-York. London: Academic Press 1978

4. Aliprantis, C.D.. Burkinshaw, O.: Positive Operators. New York. London: Academic Press 1985

5. Back, K.: Structure of consumption sets and existence of equilibria in infinite-dimensional spaces. J. Math. Econom.17,89-99 (1988)

6. Balasko, Y., Cass, D., Shel1, K.: Existence of competitive equilibrium in a general overlapping-generations model, J.Econ. Theory 23, 307-322 (1980)

7. Balasko, Y., Shel1, K.: The overlapping-generations model, I : The case of pure exchange without money, J. Econ. Theory 23, 281-306 (1980)

8. Besada, M., Esteves, M.. Herves, C.: Equilibria in economies with countably many commodities, Econ. Lett. 26, 203-207 (1988)

9. Bergstrom, T.C.: Private communication(1991)

10. Bewley, T.F.: Existence of equilibria in economies with infinitely many commodities, J. Econ.Theory 4,514-540 (1972)

11. Burke. J.: On the existence of price equilibria in dynamic economies, J. Econ. Theory 44, 281-300 (1988)

12. Cherif. I.: Existence d'un équilibre pour une économie avec une infinite dénombrable de consommateurs. Application au modele a genérations. Mémoire de DEA: Universite de Pario I-Ecole polytechnique 1990 
13. Cherif, I., Florenzano. M.: Existence de lequilibre dans le modele a genérations. Working-paper CEPREMAP n*9116 (1991)

14. Florenzano, M.: Edgeworth equilibria, fuzzy core and equilibria of a production economy without ordered preferences, J. Math. Anal. Appl. 153. $18-36(1990)$

15. Florenzano, M.: Markets with countably many periods. Working-paper CEPREMAP $\mathrm{B}^{\cdot} 9019(1990)$

16. Geanakoplos, J.D., Polemarchakis, H.M.: Overlapping generations. In: Hildenbrand, W.. Sonnenschein, H. (eds) Handbook of Mathematical Economics, vo1. Iv, 1899-1960. Amsterdam: North-Holland 1991

17. Kothe. G.: Topological Vector Spaces I. Berlin, Heidelberg. New York: Springer 1969

18. Mas-colel1. A.: The price equilibrium existence problem in topological vector lattices, Econometrica 54, 1039-1053 (1986)

19. Richard, S.F., Srivastava, S.: Equilibrium in economies with infinitely many consumers and infinitely many comodities. J. Math. Econ. 17, 9-21 (1988)

20. Samuelion. P.A.: An exact consumption-loan model of interest with or without the social contrivance of money, J. Polit. Econ. 66,467-482 (1958)

21. Wilson, C.A.: Equilibrium in dynamic models with an infinity of agents. J. Econ. Theory 24.95-111(1981)

22. Yannelis, N.C.. Zame, W.R.: Equilibria in Banach lattices without ordered preferences. J. Math. Econ. 15, 85-110 (1986)

23. Zame, W.R.: Equilibria in production economies with an infinite dimensional commodity space, Econometrica 55, n'5, 1075-1108(1987) 